\title{
Numerical Methods for Fractional Order Singular Partial Differential Equations with Variable Coefficients
}

\author{
Asma Ali Elbeleze, ${ }^{1,2}$ Adem Kılıçman, ${ }^{3}$ and Bachok M. Taib ${ }^{1}$ \\ ${ }^{1}$ Faculty of Science and Technology, Universiti Sains Islam Malaysia, 71800 Nilai, Malaysia \\ ${ }^{2}$ Department of Mathematics, Zawia University, Zawia, Libya \\ ${ }^{3}$ Department of Mathematics and Institute of Mathematical Research, University Putra Malaysia, 4300 Serdang, Selangor, Malaysia
}

Correspondence should be addressed to Adem Kılıçman; akilic@upm.edu.my

Received 11 October 2013; Accepted 22 January 2014; Published 10 March 2014

Academic Editor: Abdon Atangana

Copyright (C) 2014 Asma Ali Elbeleze et al. This is an open access article distributed under the Creative Commons Attribution License, which permits unrestricted use, distribution, and reproduction in any medium, provided the original work is properly cited.

\begin{abstract}
We implement relatively analytical methods, the homotopy perturbation method and the variational iteration method, for solving singular fractional partial differential equations of fractional order. The process of the methods which produce solutions in terms of convergent series is explained. The fractional derivatives are described in Caputo sense. Some examples are given to show the accurate and easily implemented of these methods even with the presence of singularities.
\end{abstract}

\section{Introduction}

In the last decades, fractional calculus found many applications in various fields of engineering and physical sciences such as physics, chemistry, biology, economy, viscoelasticity, electrochemistry, electromagnetic, relaxation processes, diffusion, control, porous media, and many more; see, for example, [1-6].

The numerical solution of singular differential equations of integer order has been a hot topic in numerical and computational mathematics for a long time $[7,8]$. Singular partial differential equations of fractional order, as generalizations of classical singular partial differential equations of integer order, are increasingly used to model problems in physics and engineering. Consequently, considerable attention has been given to the solution of singular partial differential equations of fractional order.

In most of these equations analytical solutions are either quite difficult or impossible to achieve, so approximations and numerical techniques must be used. Several methods have been used to solve singular differential equations such as variational iteration method [8], homotopy perturbation method [7], and homotopy analysis method [9].

The homotopy perturbation method [10-12] and variational iteration method [13-16] are relatively new approach to provide an analytical approximation to linear and nonlinear problems, and they are particularly valuable as tools for scientists and applied mathematicians, because it provides immediate and visible symbolic terms of analytic solutions, as well as numerical approximate solutions to both linear and nonlinear differential equations.

Recently, the application of these methods extended for fractional differential equations. He [15] was the first to apply the variational iteration method to fractional differential equations.

The objective of the present paper is to extend the application of homotopy perturbation method to provide approximate solutions and to make comparison with that obtained by the variational iteration method for singular partial differential equations of fractional order:

$$
\begin{array}{r}
\frac{\partial^{\alpha} u}{\partial t^{\alpha}}+\mu(x) \frac{\partial^{4} u}{\partial x^{4}}+\lambda(y) \frac{\partial^{4} u}{\partial y^{4}}+h(z) \frac{\partial^{4} u}{\partial z^{4}}=0, \\
a<x, y, z<b, \quad t>0,
\end{array}
$$

where $\mu(x), \lambda(y), h(z)$ subject to initial conditions

$$
u(x, y, z, 0)=f_{0}(x, y, z), \quad \frac{\partial u}{\partial t}(x, y, z, 0)=f_{1}(x, y, z)
$$


and boundary conditions

$$
\begin{aligned}
& u(a, y, z, t)=g_{0}(y, z, t), \\
& u(b, y, z, t)=g_{1}(y, z, t), \\
& u(x, a, z, t)=g_{2}(x, z, t), \\
& u(x, b, z, t)=g_{3}(x, z, t), \\
& u(x, y, a, t)=g_{4}(x, z, t), \\
& u(x, y, b, t)=g_{5}(x, z, t), \\
& \frac{\partial^{2} u}{\partial x^{2}}(a, y, z, t)=k_{0}(y, z, t), \\
& \frac{\partial^{2} u}{\partial x^{2}}(b, y, z, t)=k_{0}(y, z, t), \\
& \frac{\partial^{2} u}{\partial y^{2}}(x, a, z, t)=k_{2}(x, z, t), \\
& \frac{\partial^{2} u}{\partial y^{2}}(x, a, z, t)=k_{3}(x, z, t), \\
& \frac{\partial^{2} u}{\partial z^{2}}(x, y, a, t)=k_{4}(x, y, t), \\
& \frac{\partial^{2} u}{\partial z^{2}}(x, y, b, t)=k_{5}(y, z, t),
\end{aligned}
$$

where $\partial^{\alpha}(\cdot) / \partial t^{\alpha}$ is the fractional derivative in the Caputo sense, $1<\alpha \leq 2$, and $g_{i}$ and $k_{i}, i=0, \ldots, 5$ are continuous. The paper is organized as follows. In Section 2, some basic definitions and properties of fractional calculus theory are given. In Section 3, the basic idea of HPM is given. In Section 4, the basic idea of variational iteration method is given. In Sections 5 and 6 the analysis of HPM and VIM for singular fractional partial differential equations, respectively, is given. Some examples are given in Section 7. Concluding remarks are listed in Section 8.

\section{Preliminaries}

In this section, we give some basic definitions and properties of fractional calculus theory which is used in this paper.

Definition 1. A real function $f(x), x>0$ is said to be in space $C \mu, \mu \in R$ if there exists a real number $p>\mu$, such that $f(x)=$ $x^{p} f_{1}(x)$ where $f_{1}(x) \in C(0, \infty)$, and it is said to be in the space $C_{\mu}^{n}$ if $f^{n} \in R_{\mu}, n \in N$.

Definition 2. The Riemann-Liouville fractional integral operator of order $\alpha \geq 0$ of a function $f \in C \mu, \mu \geq-1$ is defined as

$$
J^{\alpha} f(x)=\frac{1}{(\Gamma(\alpha)} \int_{0}^{x}(x-t)^{\alpha-1} f(t) d t, \quad \alpha>0, t>0
$$

in particular $J^{0} f(x)=f(x)$.
For $\beta \geq 0$ and $\gamma \geq-1$, some properties of the operator $J^{\alpha}$

(i) $J^{\alpha} J^{\beta} f(x)=J^{\alpha+\beta} f(x)$,

(ii) $J^{\alpha} J^{\beta} f(x)=J^{\beta} J^{\alpha} f(x)$,

(iii) $J^{\alpha} x^{\gamma}=(\Gamma(\gamma+1) / \Gamma(\alpha+\gamma+1)) x^{\alpha+\gamma}$.

Definition 3. The Caputo fractional derivative of $f \in C_{-1}^{m}$, $m \in N$ is defined as

$$
\begin{array}{r}
D^{\alpha} f(x)=\frac{1}{\Gamma(m-\alpha)} \int_{0}^{x}(x-t)^{m-\alpha-1} f^{m}(t) d t, \\
m-1<\alpha \leq m .
\end{array}
$$

Lemma 4. if $m-1<\alpha \leq m, m \in N, f \in C_{\mu}^{m}, \mu>-1$ then the following two properties hold:

(i) $D^{\alpha}\left[J^{\alpha} f(x)\right]=f(x)$,

(ii) $J^{\alpha}\left[D^{\alpha} f(x)\right]=f(x)-\sum_{k=1}^{m-1} f^{k}(0)\left(x^{k} / k !\right)$.

\section{Homotopy Perturbation Method}

To illustrate the basic idea of this method, we consider the following nonlinear differential equation:

$$
A(u)-f(r)=0, \quad r \in \Omega,
$$

with boundary conditions

$$
B\left(u, \frac{\partial u}{\partial n}\right)=0, \quad r \in \Gamma,
$$

where $A$ is a general differential operator, $B$ is a boundary operator, $f(r)$ is a known analytic function, and $\Gamma$ is the boundary of the domain $\Omega$.

In general, the operator $A$ can be divided into two parts $L$ and $N$, where $L$ is linear, while $N$ is nonlinear. Equation (6) therefore can be rewritten as follows:

$$
L(u)+N(u)-f(r)=0 .
$$

By the homotopy technique $[10,11]$, we construct a homotopy $v(r, p): \Omega \times[0,1] \rightarrow R$ which satisfies

$$
\begin{array}{r}
H(v, p)=(1-p)\left[L(v)-L\left(u_{0}\right)\right]+p[A(v)-f(r)]=0 \\
p \in[0,1], \quad r \in \Omega
\end{array}
$$

or

$$
H(v, p)=L(v)-L\left(u_{0}\right)+p L\left(u_{0}\right)+p[N(v)-f(r)]=0,
$$

where $p \in[0,1]$ is an embedding parameter and $u_{0}$ is an initial approximation of (6) which satisfies the boundary conditions.

From (9) and (10) we have

$$
\begin{aligned}
& H(v, 0)=L(v)-L\left(u_{0}\right)=0, \\
& H(v, 1)=A(v)-f(r)=0 .
\end{aligned}
$$


The change in the process of $p$ from zero to unity is just that of $v(r, p)$ from $u_{0}(r)$ to $u(r)$. In topology this is called deformation and $L(v)-L\left(u_{0}\right)$ and $A(v)-f(r)$ are called homotopic.

Now, assume that the solution of (9) and (10) can be expressed as

$$
v=v_{0}+p v_{1}+p^{2} v_{2}+\cdots
$$

Setting $p=1$ results in the approximate solution of (6). Consider

$$
u=\lim _{p \rightarrow 1} v=v_{0}+v_{1}+v_{2}+\cdots
$$

\section{The Variational Iteration Method}

To illustrate the basic concepts of VIM, we consider the following differential equation:

$$
L(u)+N(u)=g(x) \text {, }
$$

where $L$ is a linear operator, $N$ is a nonlinear operator, and $g(x)$ is a nonhomogeneous term.

According to VIM, one constructs a correction functional as follows:

$$
y_{n+1}=y_{n}+\int_{0}^{x} \lambda\left[L y_{n}(s)-N \widetilde{y_{n}}(s)\right] d s,
$$

where $\lambda$ is a general Lagrange multiplier, and $\widetilde{y_{n}}$ denotes restricted variation; that is, $\delta \overline{y_{n}}=0$.

\section{Analysis of Homotopy Perturbation Method}

To illustrate the basic concepts of HPM for (1) with (2) and (3), we use the view of $\mathrm{He}[10,11]$, where the following homotopy was constructed for (1):

$$
\begin{aligned}
& (1-p) \frac{\partial^{\alpha} u}{\partial t^{\alpha}} \\
& +p\left(\frac{\partial^{\alpha} u}{\partial t^{\alpha}}+\mu(x) \frac{\partial^{4} u}{\partial x^{4}}+\lambda(y) \frac{\partial^{4} u}{\partial y^{4}}+h(z) \frac{\partial^{4} u}{\partial z^{4}}\right)=0
\end{aligned}
$$

or

$$
\frac{\partial^{\alpha} u}{\partial t^{\alpha}}+p\left(\mu(x) \frac{\partial^{4} u}{\partial x^{4}}+\lambda(y) \frac{\partial^{4} u}{\partial y^{4}}+h(z) \frac{\partial^{4} u}{\partial z^{4}}\right)=0,
$$

where $p \in[0,1]$ is an embedding parameter. If $p=0$, (17) becomes linear fractional differential equation

$$
\frac{\partial^{\alpha} u}{\partial t^{\alpha}}=0
$$

and when $p=1$, (17) turns out to be the original equation. In view of basic assumption of HPM, solution of (1) can be expressed as a power series in $p$

$$
u(x, t)=u_{0}(x, t)+p_{1} u_{1}(x, t)+p_{2} u_{2}(x, t)+\cdots .
$$

When $p=1$, we get the approximate solution of (1)

$$
u(x, t)=u_{0}(x, t)+u_{1}(x, t)+u_{2}(x, t)+\cdots .
$$

The convergence of series (20) has been proved in [12].

Substituting (19) into (17) and then equating the terms with identical power of $p$, we obtain the following series of linear equations:

$$
\begin{gathered}
p^{0}: \frac{\partial^{\alpha} u_{0}}{\partial t^{\alpha}}=0 \\
p^{1}: \frac{\partial^{\alpha} u_{1}}{\partial t^{\alpha}}=\mu(x) \frac{\partial^{4} u_{0}}{\partial x^{4}}+\lambda(y) \frac{\partial^{4} u_{0}}{\partial y^{4}}+h(z) \frac{\partial^{4} u_{0}}{\partial z^{4}} \\
p^{2}: \frac{\partial^{\alpha} u_{2}}{\partial t^{\alpha}}=\mu(x) \frac{\partial^{4} u_{1}}{\partial x^{4}}+\lambda(y) \frac{\partial^{4} u_{1}}{\partial y^{4}}+h(z) \frac{\partial^{4} u_{1}}{\partial z^{4}} \\
p^{3}: \frac{\partial^{\alpha} u_{3}}{\partial t^{\alpha}}=\mu(x) \frac{\partial^{4} u_{2}}{\partial x^{4}}+\lambda(y) \frac{\partial^{4} u_{2}}{\partial y^{4}}+h(z) \frac{\partial^{4} u_{2}}{\partial z^{4}}
\end{gathered}
$$

with the initial conditions (2) and boundary conditions (3).

The initial approximation can be chosen in the following manner.

$$
\begin{aligned}
& u_{0}=\sum_{j=0}^{1} \gamma_{j} \frac{t^{j}}{j !}=\gamma_{0}+\gamma_{1} t, \\
& \text { where } \gamma_{0}=f_{0}(x, y, z), \gamma_{1}=f_{1}(x, y, z) .
\end{aligned}
$$

The equations in the system (21) can be solved by applying the operator $J_{*}^{\alpha}$ and by simple computation; we approximate the series solution of HPM by the following N-term truncated series:

$$
u(x, t)=u_{0}(x, t)+u_{1}(x, t)+\cdots+u_{n}(x, t),
$$

which is the approximate solution of (1) with (2) and (3).

\section{Analysis of Variational Iteration Method}

To solve the fractional singular partial differential equations by using the variational iteration method, with initial and boundary conditions (2) and (3), we construct the following correction functional:

$$
u_{k+1}(x, t)=u_{k}(x, t)+J_{t}^{\alpha}\left[\lambda\left(\frac{\partial^{\alpha} u_{k}(x, t)}{\partial t^{\alpha}}+\mathbf{R} \widetilde{u}_{k}(x, t)\right)\right],
$$

or

$$
\begin{aligned}
u_{k+1} & (x, t) \\
= & u_{k}(x, t)+\frac{1}{\Gamma(\alpha)} \delta \\
& \quad \times \int_{0}^{t}(t-s)^{\alpha-1} \lambda(s)\left(\frac{\partial^{\alpha} u_{k}(x, s)}{\partial s^{\alpha}}+\mathbf{R} \tilde{u}_{k}(x, s)\right) d s,
\end{aligned}
$$


where

$$
\begin{aligned}
\mathbf{R} \tilde{u}_{k}(x, s)= & \mu(x) \frac{\partial^{4} \tilde{u}_{k}(x, s)}{\partial x^{4}}+\lambda(y) \frac{\partial^{4} \tilde{u}_{k}(x, s)}{\partial y^{4}} \\
& +h(z) \frac{\partial^{4} \tilde{u}_{k}(x, s)}{\partial z^{4}},
\end{aligned}
$$

and $J_{t}^{\alpha}$ is the Riemann-Liouville fractional integral operator of order $\alpha$, with respect to variable $t, \lambda$ is a general Lagrange multiplier which can be identified optimally by variational theory [17], and $\tilde{u}_{k}(x, t)$ are considered as restricted variation; that is, $\delta \widetilde{u}_{k}(x, t)=0$.

Making the above correction functional stationary, the following condition can be obtained:

$$
\begin{aligned}
& \delta u_{k+1}(x, t) \\
& =\delta u_{k}(x, t)+\frac{1}{\Gamma(\alpha)} \delta \\
& \quad \times \int_{0}^{t}(t-s)^{\alpha-1} \lambda(s)\left(\frac{\partial^{\alpha} u_{k}(x, s)}{\partial s^{\alpha}}+\mathbf{R} \tilde{u}_{k}(x, s)\right) d s
\end{aligned}
$$

which yields to Lagrange multiplier

$$
\lambda(s)=s-t
$$

We obtain the following iteration formula by substitution of (28) in (25):

$$
\begin{aligned}
u_{k+1}(x, t) & \\
= & u_{k}(x, t)+\frac{1}{\Gamma(\alpha-1)} \\
& \times \int_{0}^{t}(t-s)^{\alpha-2}(t-s)\left(\frac{\partial^{\alpha} u_{k}(x, s)}{\partial s^{\alpha}}+R u_{k}(x, s)\right) d s .
\end{aligned}
$$

That is,

$$
\begin{aligned}
u_{k+1}(x, t)= & u_{k}(x, t)-\frac{(\alpha-1)}{\Gamma(\alpha)} \\
& \times \int_{0}^{t}(t-s)^{\alpha-1}\left(\frac{\partial^{\alpha} u_{k}(x, s)}{\partial s^{\alpha}}+R u_{k}(x, s)\right) d s
\end{aligned}
$$

This yields the following iteration formula:

$$
\begin{aligned}
u_{k+1}(x, t)= & u_{k}(x, t)-(\alpha-1) J_{t}^{\alpha} \\
& \times\left(\frac{\partial^{\alpha} u_{k}(x, t)}{\partial t^{\alpha}}+R u_{k}(x, t)\right) .
\end{aligned}
$$

The initial approximation $u_{0}$ can be chosen by the following manner which satisfies initial conditions:

$$
u_{0}=\sum_{j=0}^{1} \gamma_{j} \frac{t^{j}}{j !}=\gamma_{0}+\gamma_{1} t
$$

where $\gamma_{0}=f_{0}(x, y, z), \gamma_{1}=f_{1}(x, y, z)$.
We can obtain the following first-order approximation by substitution of (32) in (31):

$$
u_{1}(x, t)=u_{0}(x, t)-(\alpha-1) J_{t}^{\alpha}\left(\frac{\partial^{\alpha} u_{0}(x, t)}{\partial t^{\alpha}}+R u_{0}(x, t)\right) \text {. }
$$

Finally, by substituting the constant values of $\gamma_{0}$ and $\gamma_{1}$ in (33), we have the results as the approximate solutions of (1) with (2) and (3).

\section{Applications}

In this section we have applied homotopy perturbation method and variational iteration method to fractional singular partial differential equations with known exact solution.

Example 5. Consider the following fourth-order fractional singular partial differential equation:

$$
\begin{array}{r}
\frac{\partial^{\alpha} u}{\partial t^{\alpha}}+\left(\frac{x}{\sin x}-1\right) \frac{\partial^{4} u}{\partial x^{4}}=0, \\
0<x<1, \quad t>0, \quad 1<\alpha \leq 2 .
\end{array}
$$

With initial conditions

$$
\begin{gathered}
u(x, 0)=x-\sin x, \quad 0<x<1 \\
\frac{\partial u}{\partial t}(x, 0)=-(x-\sin x), \quad 0<x<1
\end{gathered}
$$

and with boundary conditions

$$
\begin{aligned}
& u(0, t)=0, \quad u(1, t)=e^{-t}(1-\sin 1), \quad t>0 \\
& \frac{\partial^{2} u}{\partial x^{2}}(0, t)=0, \quad \frac{\partial^{2} u}{\partial x^{2}}(1, t)=e^{-t} \sin 1, \quad t>1 .
\end{aligned}
$$

The exact solution in special case $\alpha=2$ is

$$
u(x, t)=(x-\sin x) e^{-t} .
$$

First, we construct

$$
\frac{\partial^{\alpha} u}{\partial t^{\alpha}}=-p\left(\frac{x}{\sin x}-1\right) \frac{\partial^{4} u}{\partial x^{4}} .
$$

Substituting (19) in (38) and then equating the terms with same powers of $p$, we get the series

$$
\begin{gathered}
p^{0}: \frac{\partial^{\alpha} u_{0}}{\partial t^{\alpha}}=0, \quad u_{0}(x, t)=x-\sin x \\
\frac{\partial u_{0}}{\partial t}(x, 0)=-(x-\sin x) \\
p^{1}: \frac{\partial^{\alpha} u_{1}}{\partial t^{\alpha}}=-\left(\frac{x}{\sin x}-1\right) \frac{\partial^{4} u_{0}}{\partial x^{4}} \\
p^{2}: \frac{\partial^{\alpha} u_{2}}{\partial t^{\alpha}}=-\left(\frac{x}{\sin x}-1\right) \frac{\partial^{4} u_{1}}{\partial x^{4}}
\end{gathered}
$$


Now applying the operator $J_{t}^{\alpha}$ to (39) and using initial and boundary conditions yield

$$
\begin{gathered}
u_{0}(x, t)=(x-\sin x)-(x-\sin x) t \\
u_{1}(x, t)=(x-\sin x) \frac{t^{\alpha}}{\Gamma(\alpha+1)}-(x-\sin x) \frac{t^{\alpha+1}}{\Gamma(\alpha+2)}, \\
u_{2}(x, t)=(x-\sin x) \frac{t^{2 \alpha}}{\Gamma(2 \alpha+1)}-(x-\sin x) \frac{t^{2 \alpha+1}}{\Gamma(2 \alpha+2)},
\end{gathered}
$$

so that the solution $u(x, t)$ of the problem (34) is given by

$$
\begin{aligned}
u(x, t) & =u_{0}(x, t)+u_{1}(x, t)+u_{2}(x, t)+\cdots \\
u(x, t)= & (x-\sin x)-(x-\sin x) t+(x-\sin x) \\
& \times \frac{t^{\alpha}}{\Gamma(\alpha+1)}-(x-\sin x) \frac{t^{\alpha+1}}{\Gamma(\alpha+2)} \\
& +(x-\sin x) \frac{t^{2 \alpha}}{\Gamma(2 \alpha+1)} \\
& -(x-\sin x) \frac{t^{2 \alpha+1}}{\Gamma(2 \alpha+2)}+\cdots
\end{aligned}
$$

Now, we solve by variational iteration method. According to variational iteration method, the formula (31) for (34) can be expressed in the following form:

$$
\begin{aligned}
u_{k+1}(x, t)= & u_{k}(x, t)-(\alpha-1) J_{t}^{\alpha} \\
& \times\left(\frac{\partial^{\alpha} u_{k}(x, t)}{\partial t^{\alpha}}+\left(\frac{x}{\sin x}-1\right) \frac{\partial^{4} u}{\partial x^{4}}\right) .
\end{aligned}
$$

Suppose that an initial approximation has the following form which satisfies the initial conditions:

$$
u_{0}(x, t)=(x-\sin x)-(x-\sin x) t
$$

Now by iteration formula (42), we obtain the first approximation:

$$
\begin{aligned}
u_{1}(x, t)= & u_{0}(x, t)-(\alpha-1) J_{t}^{\alpha} \\
& \times\left(\frac{\partial^{\alpha} u_{0}(x, t)}{\partial t^{\alpha}}+\left(\frac{x}{\sin x}-1\right) \frac{\partial^{4} u_{0}}{\partial x^{4}}\right) \\
= & (x-\sin x)-(x-\sin x) t+(\alpha-1)(x-\sin x) \\
& \times \frac{t^{\alpha}}{\Gamma(\alpha+1)}-(\alpha-1)(x-\sin x) \frac{t^{\alpha+1}}{\Gamma(\alpha+2)}
\end{aligned}
$$

and second approximation:

$$
\begin{aligned}
u_{2}(x, t)= & u_{1}(x, t)-(\alpha-1) J_{t}^{\alpha} \\
& \times\left(\frac{\partial^{\alpha} u_{1}(x, t)}{\partial t^{\alpha}}+\left(\frac{x}{\sin x}-1\right) \frac{\partial^{4} u_{1}}{\partial x^{4}}\right) \\
= & (x-\sin x)-(x-\sin x) t \\
& -(\alpha-3)(\alpha-1)(x-\sin x) \frac{t^{\alpha}}{\Gamma(\alpha+1)} \\
& +(\alpha-3)(\alpha-1)(x-\sin x) \frac{t^{\alpha+1}}{\Gamma(\alpha+2)} \\
& +(\alpha-1)^{2}(x-\sin x) \frac{t^{2 \alpha}}{\Gamma(2 \alpha+1)} \\
& -(\alpha-1)^{2}(x-\sin x) \frac{t^{2 \alpha+1}}{\Gamma(2 \alpha+2)} .
\end{aligned}
$$

Table 1 shows the approximate solution for (34) obtained for different values of $\alpha$ using the homotopy perturbation method and the variational iteration method. The value $\alpha=$ 2 is the only case for which we know the exact solution $u(x, t)=(x-\sin x) e^{-t}$ and our approximate solution using the variational iteration method is more accurate than approximate solution obtained using homotopy perturbation method. It is to be noted that only the third-order term series was used in evaluating the approximate solutions for Table 1.

Example 6. Consider the following singular two-dimensional partial differential equation of fractional order:

$$
\begin{array}{r}
\frac{\partial^{\alpha} u}{\partial t^{\alpha}}+2\left(\frac{1}{x^{2}}+\frac{x^{4}}{6 !}\right) \frac{\partial^{4} u}{\partial x^{4}}+2\left(\frac{1}{y^{2}}+\frac{y^{4}}{6 !}\right) \frac{\partial^{4} u}{\partial y^{4}} \\
0<x, \quad y<1, \quad t>0, \quad 1<\alpha \leq 2 .
\end{array}
$$

With initial conditions

$$
\begin{gathered}
u(x, y, 0)=0, \quad 0<x<1 \\
\frac{\partial u}{\partial t}(x, y, 0)=2+\frac{x^{6}}{6 !}+\frac{y^{6}}{6 !}, \quad 0<x<1
\end{gathered}
$$

and with boundary conditions

$$
\begin{gathered}
u(0.5, y, t)=\left(2+\frac{(0.5)^{6}}{6 !}+\frac{y^{6}}{6 !}\right) \sin t, \\
u(1, y, t)=\left(2+\frac{1}{6 !}+\frac{y^{6}}{6 !}\right) \sin t, \quad t>0, \\
\frac{\partial^{2} u}{\partial x^{2}}(0.5, y, t)=\frac{(0.5)^{4}}{6 !} \sin t \\
\frac{\partial^{2} u}{\partial x^{2}}(1, y, t)=\frac{1}{6 !} \sin t, \quad t>1, \\
\frac{\partial^{2} u}{\partial y^{2}}(x, 0.5, t)=\frac{(0.5)^{4}}{6 !} \sin t, \\
\frac{\partial^{2} u}{\partial y^{2}}(x, 1, t)=\frac{1}{6 !} \sin t, \quad t>1,
\end{gathered}
$$


TABLE 1: Numerical values when $\alpha=1.5,1.75$, and 2.0 for (34).

\begin{tabular}{|c|c|c|c|c|c|c|c|}
\hline \multirow{2}{*}{$x$} & \multicolumn{2}{|c|}{$\alpha=1.5$} & \multicolumn{2}{|c|}{$\alpha=1.75$} & \multicolumn{3}{|c|}{$\alpha=2$} \\
\hline & $u_{\mathrm{HPM}}$ & $u_{\mathrm{VIM}}$ & $u_{\mathrm{HPM}}$ & $u_{\mathrm{VIM}}$ & $u_{\mathrm{HPM}}$ & $u_{\mathrm{VIM}}$ & $u_{\text {Exact }}$ \\
\hline 0.1 & 0.000144 & 0.000141 & 0.000139 & 0.000139 & 0.000136 & 0.000136 & 0.000136 \\
\hline 0.2 & 0.001149 & 0.001127 & 0.034155 & 0.001108 & 0.001089 & 0.001089 & 0.001089 \\
\hline 0.3 & 0.003867 & 0.003793 & 0.003740 & 0.003729 & 0.003668 & 0.003668 & 0.003667 \\
\hline 0.4 & 0.009134 & 0.008960 & 0.007492 & 0.008809 & 0.008663 & 0.008664 & 0.008664 \\
\hline 0.5 & 0.017759 & 0.017421 & 0.017175 & 0.017128 & 0.016845 & 0.016844 & 0.016845 \\
\hline 0.6 & 0.030519 & 0.029939 & 0.029516 & 0.029435 & 0.028949 & 0.028948 & 0.028948 \\
\hline 0.7 & 0.048149 & 0.047233 & 0.046566 & 0.046438 & 0.045671 & 0.045671 & 0.045671 \\
\hline 0.8 & 0.071336 & 0.069978 & 0.068989 & 0.068801 & 0.067663 & 0.067663 & 0.067663 \\
\hline 0.9 & 0.100708 & 0.098792 & 0.097396 & 0.097130 & 0.095524 & 0.095524 & 0.095524 \\
\hline
\end{tabular}

and the exact solution in special case $\alpha=2$ is

$$
u(x, y, t)=\left(2+\frac{x^{6}}{6 !}+\frac{y^{6}}{6 !}\right) \sin t
$$

According to HPM, we construct the following homotopy:

$$
\frac{\partial^{\alpha} u}{\partial t^{\alpha}}=p\left(2\left(\frac{1}{x^{2}}+\frac{x^{4}}{6 !}\right) \frac{\partial^{4} u}{\partial x^{4}}+2\left(\frac{1}{y^{2}}+\frac{y^{4}}{6 !}\right) \frac{\partial^{4} u}{\partial y^{4}}\right) \text {. }
$$

Substituting (19) in (50) and then equating the terms with same powers of $p$, we get the series

$$
\begin{gathered}
p^{0}: \frac{\partial^{\alpha} u_{0}}{\partial t^{\alpha}}=0, \\
u_{0}(x, t)=0, \quad \frac{\partial u_{0}}{\partial t}(x, 0)=2+\frac{x^{6}}{6 !}+\frac{y^{6}}{6 !} \\
p^{1}: \frac{\partial^{\alpha} u_{1}}{\partial t^{\alpha}}=-\left(2\left(\frac{1}{x^{2}}+\frac{x^{4}}{6 !}\right) \frac{\partial^{4} u_{0}}{\partial x^{4}}+2\left(\frac{1}{y^{2}}+\frac{y^{4}}{6 !}\right) \frac{\partial^{4} u_{0}}{\partial y^{4}}\right), \\
p^{2}: \frac{\partial^{\alpha} u_{2}}{\partial t^{\alpha}}=-\left(2\left(\frac{1}{x^{2}}+\frac{x^{4}}{6 !}\right) \frac{\partial^{4} u_{1}}{\partial x^{4}}+2\left(\frac{1}{y^{2}}+\frac{y^{4}}{6 !}\right) \frac{\partial^{4} u_{1}}{\partial y^{4}}\right),
\end{gathered}
$$

Now applying the operator $J_{t}^{\alpha}$ to (51) and using initial and boundary conditions yield

$$
\begin{gathered}
u_{0}(x, t)=\left(2+\frac{x^{6}}{6 !}+\frac{y^{6}}{6 !}\right) t \\
u_{1}(x, t)=-\left(2+\frac{x^{6}}{6 !}+\frac{y^{6}}{6 !}\right) \frac{t^{\alpha+1}}{\Gamma(\alpha+2)}, \\
u_{2}(x, t)=\left(2+\frac{x^{6}}{6 !}+\frac{y^{6}}{6 !}\right) \frac{t^{2 \alpha+1}}{\Gamma(2 \alpha+2)},
\end{gathered}
$$

so that the solution $u(x, t)$ of the problem (46) is given by

$$
u(x, t)=u_{0}(x, t)+u_{1}(x, t)+u_{2}(x, t)+\cdots
$$

$$
\begin{aligned}
u(x, t) & =\left(2+\frac{x^{6}}{6 !}+\frac{y^{6}}{6 !}\right) t-\left(2+\frac{x^{6}}{6 !}+\frac{y^{6}}{6 !}\right) \frac{t^{\alpha+1}}{\Gamma(\alpha+2)} \\
& +\left(2+\frac{x^{6}}{6 !}+\frac{y^{6}}{6 !}\right) \frac{t^{2 \alpha+1}}{\Gamma(2 \alpha+2)}+\cdots \\
= & \left(2+\frac{x^{6}}{6 !}+\frac{y^{6}}{6 !}\right)\left(t-\frac{t^{\alpha+1}}{\Gamma(\alpha+2)}+\frac{t^{2 \alpha+1}}{\Gamma(2 \alpha+2)}+\cdots\right) .
\end{aligned}
$$

Now, we solve the problem (46) by variational iteration method. According to variational iteration method, the formula (31) for (46) can be expressed in the following form:

$$
\begin{aligned}
u_{k+1}(x, t)= & u_{k}(x, t)-(\alpha-1) J_{t}^{\alpha} \\
& \times\left(\frac{\partial^{\alpha} u_{k}(x, t)}{\partial t^{\alpha}}+2\left(\frac{1}{x^{2}}+\frac{x^{4}}{6 !}\right) \frac{\partial^{4} u}{\partial x^{4}}\right. \\
& \left.+2\left(\frac{1}{y^{2}}+\frac{y^{4}}{6 !}\right) \frac{\partial^{4} u}{\partial y^{4}}\right) .
\end{aligned}
$$

Suppose that an initial approximation has the following form which satisfies the initial conditions

$$
u_{0}(x, t)=\left(2+\frac{x^{6}}{6 !}+\frac{y^{6}}{6 !}\right) t
$$


TABLE 2: Numerical values when $\alpha=1.5,1.75$, and 2.0 for (46).

\begin{tabular}{|c|c|c|c|c|c|c|c|}
\hline \multirow{2}{*}{$x$} & \multicolumn{2}{|c|}{$\alpha=1.5$} & \multicolumn{2}{|c|}{$\alpha=1.75$} & \multicolumn{3}{|c|}{$\alpha=2$} \\
\hline & $u_{\mathrm{HPM}}$ & $u_{\mathrm{VIM}}$ & $u_{\mathrm{HPM}}$ & $u_{\mathrm{VIM}}$ & $u_{\mathrm{HPM}}$ & $u_{\mathrm{VIM}}$ & $u_{\text {Exact }}$ \\
\hline 0.1 & 0.389368 & 0.389267 & 0.394618 & 0.391901 & 0.397339 & 0.394672 & 0.397339 \\
\hline 0.2 & 0.389368 & 0.389927 & 0.394618 & 0.391901 & 0.397339 & 0.394672 & 0.397339 \\
\hline 0.3 & 0.389368 & 0.389268 & 0.394618 & 0.391902 & 0.397339 & 0.394672 & 0.397339 \\
\hline 0.4 & 0.389370 & 0.389270 & 0.394620 & 0.391904 & 0.397341 & 0.394674 & 0.397339 \\
\hline 0.5 & 0.389376 & 0.389276 & 0.394627 & 0.391910 & 0.397347 & 0.394681 & 0.397341 \\
\hline 0.6 & 0.389393 & 0.389293 & 0.394644 & 0.391927 & 0.397364 & 0.394698 & 0.397347 \\
\hline 0.7 & 0.389432 & 0.389332 & 0.394682 & 0.391965 & 0.397404 & 0.394736 & 0.397344 \\
\hline 0.8 & 0.389511 & 0.389409 & 0.394762 & 0.392044 & 0.397483 & 0.394816 & 0.397483 \\
\hline 0.9 & 0.389655 & 0.389555 & 0.394991 & 0.392192 & 0.397619 & 0.394963 & 0.397632 \\
\hline
\end{tabular}

Now by iteration formula (42), we obtain the following approximations:

$$
\begin{aligned}
& u_{1}(x, t) \\
& =u_{0}(x, t)-(\alpha-1) J_{t}^{\alpha} \\
& \times\left(\frac{\partial^{\alpha} u_{0}(x, t)}{\partial t^{\alpha}}+2\left(\frac{1}{x^{2}}+\frac{x^{4}}{6 !}\right) \frac{\partial^{4} u_{0}}{\partial x^{4}}\right. \\
& \left.\quad+2\left(\frac{1}{y^{2}}+\frac{y^{4}}{6 !}\right) \frac{\partial^{4} u_{0}}{\partial y^{4}}\right) \\
& =\left(2+\frac{x^{6}}{6 !}+\frac{y^{6}}{6 !}\right) t-(\alpha-1)\left(2+\frac{x^{6}}{6 !}+\frac{y^{6}}{6 !}\right) \frac{t^{\alpha+1}}{\Gamma(\alpha+2)} .
\end{aligned}
$$

The second approximation takes the following form:

$$
\begin{aligned}
u_{2}(x, t) & \\
= & u_{1}(x, t)-(\alpha-1) J_{t}^{\alpha} \\
& \times\left(\frac{\partial^{\alpha} u_{1}(x, t)}{\partial t^{\alpha}}+2\left(\frac{1}{x^{2}}+\frac{x^{4}}{6 !}\right) \frac{\partial^{4} u_{1}}{\partial x^{4}}\right. \\
& \left.\quad+2\left(\frac{1}{y^{2}}+\frac{y^{4}}{6 !}\right) \frac{\partial^{4} u_{1}}{\partial y^{4}}\right) \\
= & \left(2+\frac{x^{6}}{6 !}+\frac{y^{6}}{6 !}\right) t-2(\alpha-1)\left(2+\frac{x^{6}}{6 !}+\frac{y^{6}}{6 !}\right) \\
& \times \frac{t^{\alpha+1}}{\Gamma(\alpha+2)}+(\alpha-1)^{2}\left(2+\frac{x^{6}}{6 !}+\frac{y^{6}}{6 !}\right) \frac{t^{2 \alpha+1}}{\Gamma(2 \alpha+1)} \\
= & \left(2+\frac{x^{6}}{6 !}+\frac{y^{6}}{6 !}\right) \\
& \times\left(t-2(\alpha-1) \frac{t^{\alpha+1}}{\Gamma(\alpha+2)}+(\alpha-1)^{2} \frac{t^{2 \alpha+1}}{\Gamma(2 \alpha+1)}\right) .
\end{aligned}
$$

Table 2 shows the approximate solution for (46) obtained for different values of $\alpha$ using the homotopy perturbation method and the variational iteration method. The value $\alpha=2$ is the only case for which we know the exact solution $u(x, t)=$ $\left(2+x^{6} / 6 !+y^{6} / 6 !\right) \sin t$, and our approximate solution using homotopy perturbation method is more accurate than approximate solution obtained using the variational iteration method. It is to be noted that only the third-order term series was used in evaluating the approximate solutions for Table 2 .

\section{Conclusion}

The essential goal of this work has been to construct an approximate solution of singular partial differential equations of fractional order. The goal has been done by using the homotopy perturbation method and the variational iteration method. The methods provide the solutions in terms of convergent series with easily computable component even with the presence of singularities. Two examples were presented in Section 7 to show the accuracy of these methods; in Example 5 it seems that the approximate solution using the variational iteration method converges faster than approximate solution using homotopy perturbation method while the approximate solution in Example 6 using homotopy perturbation method converges faster than the approximate solution obtained using the variational iteration method to the exact solution. The fact that the proposed methods solve nonlinear problems without using Adomian's polynomial can be considered as advantage of these techniques over the decomposition method.

\section{Conflict of Interests}

The authors declare that they have no conflict of interests regarding the publication of this paper.

\section{Acknowledgment}

The authors express their sincere thanks to the referees for the careful and noteworthy reading of the paper and for their very helpful suggestions that improved the paper substantially. The authors also gratefully acknowledge that this work is partially supported by the University Putra Malaysia Grant no. 5527179 . 


\section{References}

[1] K. Diethelm and A. D. Freed, "On the solution of nonlinear fractional order differential equations used in the modeling of viscoelasticity," in Scientific Computing in Chemical Engineering II-Computational Fluid Dynamics, Reaction Engineering and Molecular Properties, F. Keil, W. Mackens, H. Voss, and J. Werther, Eds., pp. 217-224, Springer, Heidelberg, Germany, 1999.

[2] R. Metzler, W. Schick, H. G. Kilian, and T. F. Nonnenmacher, "Relaxation in filled polymers: a fractional calculus approach," The Journal of Chemical Physics, vol. 103, no. 16, pp. 7180-7186, 1995.

[3] R. Hilfer, Applications of Fractional Calculus in Physics, World scientific, Singapore, 2000.

[4] K. B. Oldham and J. Spanier, "The fractional calculus," in Mathematics in Science and Engineering, vol. 198, Academic Press, San Diego, Calif, USA, 1974.

[5] I. Podlubny, "Fractional differential equation," in Mathematics in Science and Engineering, vol. 198, Academic Press, San Diego, Calif, USA, 1999.

[6] K. S. Miller and B. Ross, An Introduction to the Fractional Calculus and Fractional Differential Equations, John Wiley \& Sons, New York, NY, USA, 1993.

[7] S. T. Mohydud-Din, A. Yildirim, M. M. Hosseini, and Y. Khan, "A study on systems of variable-coefficient singular parabolic partial differential equations," World Applied Sciences Journal, vol. 10, no. 11, pp. 1321-1327, 2010.

[8] M. Abokhald, "Varational iteration method for nonlinear singular two-point boundary value problems arising in human physiology," Journal of Mathematics, vol. 2013, Article ID 720134, 4 pages, 2013.

[9] S. Abbasbsndy and E. Shivanian, "Solution of singular linear vibrational BVPs by the homotopy analysis method," Journal of Numerical Mathematics and Stochastics, vol. 1, no. 1, pp. 77-84, 2009.

[10] J. H. He, "Homotopy perturbation technique," Computer Methods in Applied Mechanics and Engineering, vol. 178, no. 3-4, pp. 257-262, 1999.

[11] J. H. He, "Coupling method of a homotopy technique and a perturbation technique for non-linear problems," International Journal of Non-Linear Mechanics, vol. 35, no. 1, pp. 37-43, 2000.

[12] J. H. He, Non-pertubation methods for strongly nonlinear problems [Dissertation], de-Verlag im Internet Gmbh, Berlin, Germany, 2006.

[13] J. H. He, "Variational iteration method for delay differential equations," Communications in Nonlinear Science and Numerical Simulation, vol. 2, no. 4, pp. 235-236, 1997.

[14] J. H. He, "Approximate solution of non linear differential equations with convolution product nonlinearities," Computer Methods in Applied Mechanics and Engineering, vol. 167, pp. 6973, 1998.

[15] J. H. He, "Approximate analytical solution for seepage flow with fractional derivatives in porous media," Computer Methods in Applied Mechanics and Engineering, vol. 167, no. 1-2, pp. 57-68, 1998.

[16] J. H. He, "Variational iteration method-a kind of non-linear analytical technique: some examples," International Journal of Non-Linear Mechanics, vol. 34, no. 4, pp. 699-708, 1999.
[17] M. Inokuti, H. Sekine, and T. Mura, "General use of the Lagrange multiplier in non-linear mathematical physics," in Variational Method in the Mechanics of Solids, S. Nemat-Nasser, Ed., pp. 156-162, Pergamon Press, Oxford, UK, 1978. 


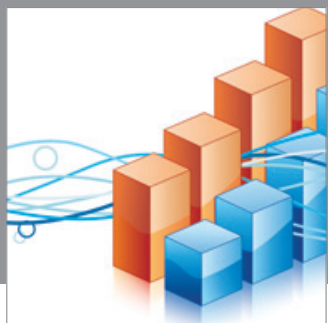

Advances in

Operations Research

mansans

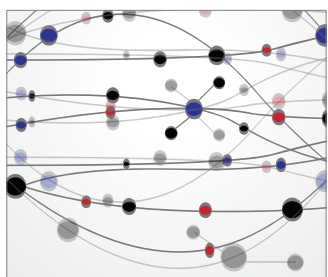

The Scientific World Journal
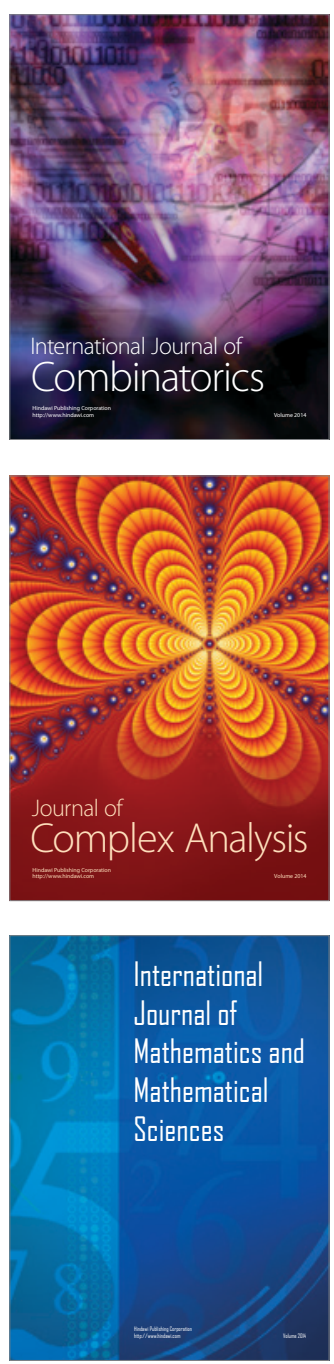
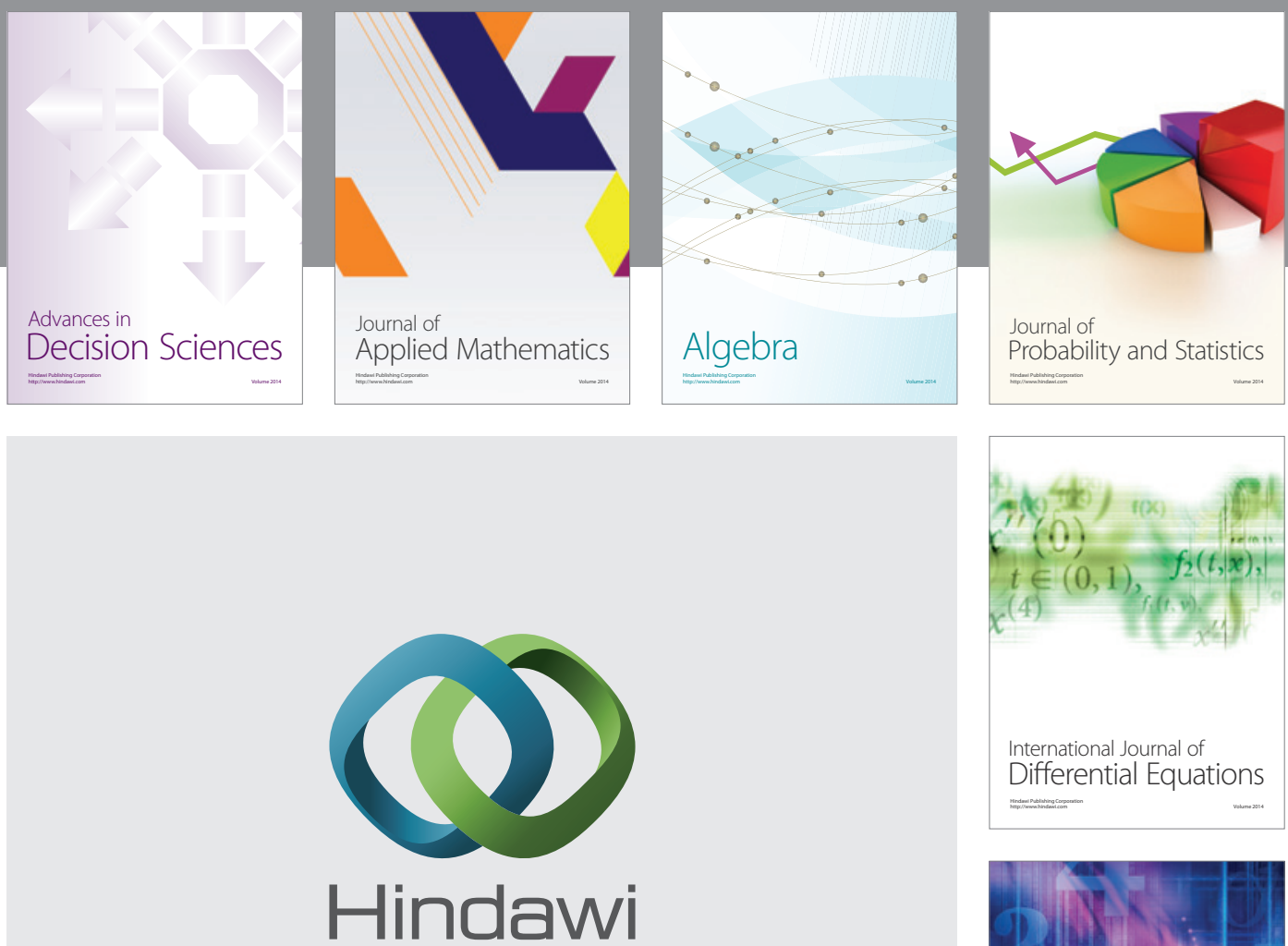

Submit your manuscripts at http://www.hindawi.com
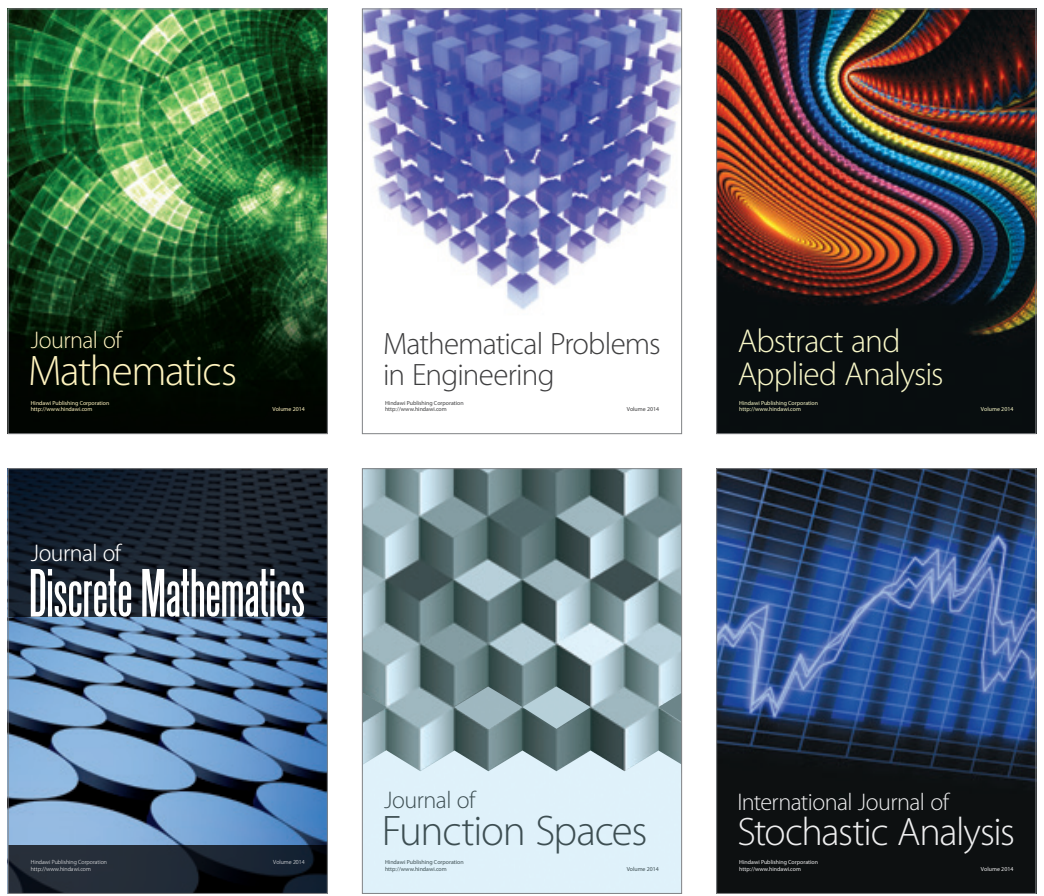

Journal of

Function Spaces

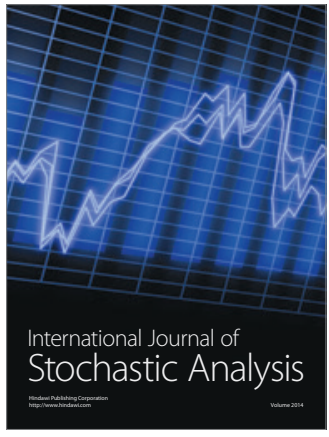

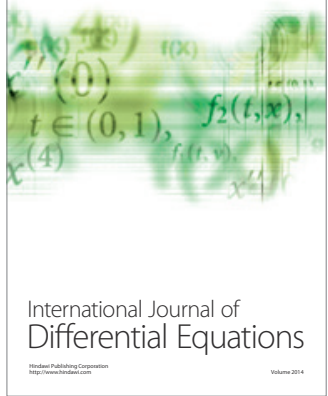
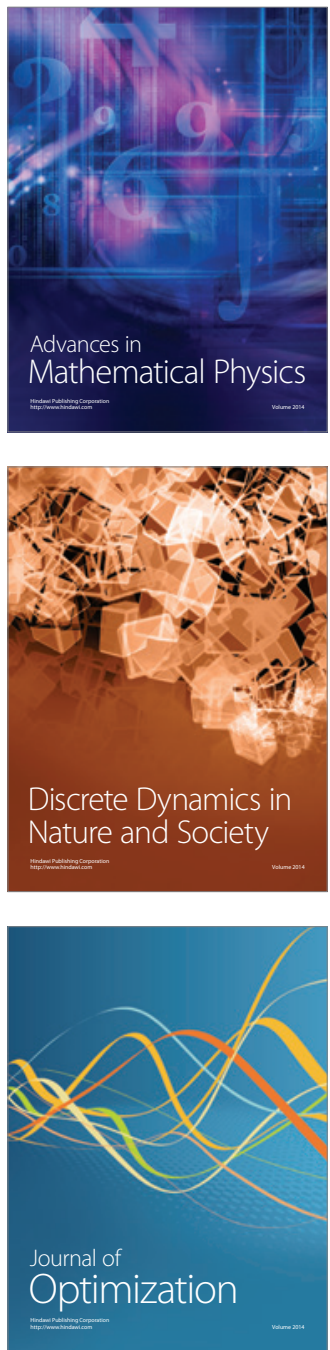